\title{
Convertir les supporters en fidèles consommateurs : une politique commerciale à Manchester United
}

\section{Claude Boli}

\section{(2) OpenEdition Journals}

Édition électronique

URL : http://journals.openedition.org/communicationorganisation/3241

DOI : 10.4000/communicationorganisation.3241

ISSN : 1775-3546

Éditeur

Presses universitaires de Bordeaux

\section{Édition imprimée}

Date de publication : 1 juin 2005

Pagination : 70-82

ISSN : $1168-5549$

\section{Référence électronique}

Claude Boli, «Convertir les supporters en fidèles consommateurs : une politique commerciale à Manchester United », Communication et organisation [En ligne], 27 | 2005, mis en ligne le 07 juin 2012, consulté le 19 avril 2019. URL : http://journals.openedition.org/communicationorganisation/3241 ; DOI : 10.4000/communicationorganisation.3241

Ce document a été généré automatiquement le 19 avril 2019.

(c) Presses universitaires de Bordeaux 


\title{
Convertir les supporters en fidèles consommateurs : une politique commerciale à Manchester United
}

\author{
Claude Boli
}

1 Depuis quelques années, selon les responsables d'instances fédérales, les dirigeants sportifs et surtout les journalistes, le club anglais de Manchester United est un cas exemplaire de la proximité entre les univers économique et sportif. Pour certaines personnes, les directions commerciales de l'équipe anglaise renvoient directement à l'expression de football-business ${ }^{1}$. En dépit de la visibilité médiatique, les observations souffrent d'une rhétorique de simplification. Il ne suffit pas de signaler que depuis le milieu des années 1990, Manchester United est le club le plus riche au monde, que le succès économique est en partie lié aux produits dérivés, et que les administrateurs affichent d'une façon ostensible des prétentions de mondialisation. Les actions qui portent sur la fidélisation des supporters permettent d'y voir plus clair, sur les raisons d'une position économique relativement dominante, dans l'espace des clubs professionnels. A condition de tenir compte d'un certain nombre de paramètres essentiels. Il faut au préalable rappeler les conditions historiques dans lesquelles se forme la politique commerciale ${ }^{2}$, afin de rompre avec les croyances selon lesquelles, tout commence à partir de l'entrée en bourse en 1991. Ensuite, nous traiterons des propriétés, notamment symboliques qui entourent un produit Manchester United. L'objet qui porte la marque Manchester United trouve auprès de certains supporters consommateurs des éléments d'identification. Enfin, en donnant la parole aux supporters nous verrons comment ils réagissent face aux mutations organisationnelles dans le club depuis ces dernières années. Comment certains endossent-ils leur nouveau rôle de supporterclient ? Et d'autres de supporter-acteur? 


\section{Historique de la politique commerciale de fidélisation}

2 La consultation des archives depuis la création de Manchester United en 1902 montre clairement, que l'inclination commerciale prend date à partir des années soixante. C'est autour des valeurs de loyauté, et de fidélité au club que s'installent les premières voies de la commercialisation. La reconstruction du stade partiellement endommagé par les bombardements de l'aviation allemande en 1941, et la volonté de reconstruire une équipe compétitive depuis les tragiques décès de joueurs de talents, lors de l'accident de Munich en 1958 obligent les administrateurs à réfléchir sur les moyens pour remplir les caisses.

3 En mai 1961, les dirigeants lancent MUDA (Manchester United Development Association). L'objectif de l'association est de collecter des fonds pour le financement de projets sportifs (recrutement de joueurs) et extra-sportifs (aménagements du stade, achat de terrain d'entraînement) $)^{3}$. L'originalité de MUDA se traduit également par l'implication des fans. La pérennité de l'organisation est aux mains des supporters, puisque ces derniers sont chargés de vendre les billets de loterie auprès de leur entourage. Le titre d'agent permet à chaque supporter d'être reconnu par les dirigeants et de démontrer l'intérêt qu'il porte à l'avenir du club. Aucune rémunération n'est attribuée à l'agent. L'engagement est perçu pour certaines personnes comme un acte de solidarité et d'affiliation. Manchester United Development Association est un succès. En 1965, la loterie rapporte $£ 34,823$ alors que la recette d'entrée au stade est de $£ 22,837$. Les sommes obtenues permettent d'entamer des rénovations dans le stade, notamment les vétustes tribunes populaires (Stretford End). Les supporters riches ne sont pas oubliés. En septembre 1965, les loges privées sont inaugurées. Les boxes sont à la mesure de l'ambition de prestige des administrateurs. A l'intérieur, sont installés un téléphone, une télévision, un bar et un système de chauffage central et un lieu de restauration avec des hôtesses. Progressivement, les exploits sportifs et le charisme de joueurs emblématiques (George Best, Denis Law) consolident la réussite des activités mercantiles. Une série de stratégies commerciales est adoptée afin de fructifier l'attachement au club des supporters. 1967 voit l'ouverture d'un magasin de souvenirs. On y trouve des produits qui permettent de renforcer l'identification à un joueur ou de glorifier une période précise. L'année 1973 est marquée par la création de Manchester United Commercial Department, organe entièrement consacré aux activités commerciales. Le service d'accueil et la restauration deviennent des préoccupations importantes. En 1985, Manchester United Basket Ball Club est fondé. A l'intérieur de United Review, la revue officielle publiée à chaque rencontre, on exhorte les supporters de football à aller encourager les basketteurs. Pour sensibiliser les fans, les directeurs évoquent la fidélité et la grandeur de Manchester United comme argument de soutien. Les années 1990 marquent un changement considérable. Des hommes recrutés pour leur expertise en matière de marketing vont grandement contribuer à l'essor économique. Avec eux, les stratégies de fidélisation connaissent une mutation profonde.

4 L'entrée en bourse en 1991 et la transformation organisationnelle que cela provoque constituent un moment décisif. Les nouvelles directions prises par la fédération de football afin d'améliorer l'image du jeu à l'échelle nationale (formation d'un nouveau championnat nommé Premier League, rénovation de stades, lutte sévère contre le hooliganisme, négociation des droits télévisés) et internationale (candidature d'organisation de compétition) favorisent les desseins des dirigeants de Manchester 
United. L'exploitation commerciale du succès sportif dans laquelle le supporter tient une place prépondérante devient une des priorités des administrateurs des Red Devils. Les perceptions sur le supporter changent radicalement. Il est perçu comme un acteur de la puissance sportive et économique. L'ambition des responsables des secteurs économiques est parfaitement délivrée dans les mots de Edward Freedman, le premier directeur du département de merchandizing: " convertir les supporters de Manchester United en véritables clients $»^{4}$. Les rapports annuels destinés aux supporters actionnaires fournissent de remarquables discours sur la philosophie commerciale. A partir de 1991, l'organigramme du club est littéralement bouleversé. Le club se singularise par sa dualité : Manchester United PLC (Public Limited Company) et Manchester United Football Club. Le premier organe a la charge des domaines économiques, et le second des questions sportives. C'est le directeur général de Centrica (fournisseur électrique), Sir Roy Gardner, dont les compétences entrepreneuriales sont régulièrement signalées dans les colonnes de la presse économique, qui est le chairman du comité de Manchester United Plc. Au sein du comité (sept membres), certains administrateurs appartiennent à la nouvelle génération de business leaders. Ils ont en commun une réputation d'experts d'entreprises hautement performants, et très médiatisés. David Gill, l'actuel directeur général est arrivé au club à l'âge de 40 ans. C'est en tant que directeur financier qu'il a construit sa réputation d'expert dans plusieurs compagnies cotées à la City, dont First Choice Holidays Plc, la puissante société d'industrie de loisirs. Philip Yea rentre dans le conseil administratif à l'âge de 44 ans. C'est le responsable des affaires de gestion. L'une de ses particularités est d'avoir été administrateur dans plusieurs sociétés anonymes européennes, d'activités non sportives, notamment Helly Hansen (Norvège) ou Moet Hennessy S.A. (France). Andy Anson, 39 ans, le nouveau directeur commercial (nommé en février 2004) est connu pour ses compétences dans l'industrie des medias. Il a notamment contribué aux succès de Channel 4 Television et Walt Disney Corporation. Les effets du recrutement de ces patrons renommés s'illustrent parfaitement dans les nouvelles politiques de rentabilité. L'utilisation du stade, comme un lieu essentiel de consommation, est une des grandes nouveautés.

5 A la différence des équipes françaises, plusieurs clubs anglais sont propriétaires de leur stade, ce qui signifie qu'ils peuvent en tirer de grands bénéfices. A Manchester United, le stade d'old Trafford ${ }^{5}$ et quelques terrains aux alentours constituent d'importantes sources financières. L'exploitation du stade, en particulier le développement d'activités commerciales, prend une tournure totalement différente des années précédentes. L'agrandissement de la capacité d'accueil devient un enjeu capital. Depuis 1995, Manchester United est le club qui investit le plus dans les aménagements de stade. Entre 1995 et 2000, 99 millions de livres sterling sont dépensés ${ }^{6}$. L'augmentation de la capacité d'accueil d'old Trafford reflète une demande de plus en plus nombreuse. La moyenne de spectateurs continue d'accroître, puisqu'elle est passée de 43681 en 1994 à 67500 en 2004. Le prestige du club à l'échelle locale permet de poursuivre le projet d'agrandissement du stade à une capacité de 75600 pour le début de la saison 2006-2007. A l'extérieur du stade, les édifications d'impressionnants superstores puis mégastores reflètent également les prétentions des dirigeants. Ici, il s'agit d'une étape importante dans les produits dérivés (verre, couvre-lit, maillot, pâte dentifrice). En 1997, l'ouverture d'un mégastore complète deux autres endroits où les supporters peuvent se procurer les objets du club. En 2000, c'est l'inauguration d'un nouveau mégastore ultra moderne de $17500 \mathrm{~m}^{2}$ situé au front de la tribune Est, entièrement rénovée. Comme il apparait dans le rapport annuel de 2004, les administrateurs ont l'intention de maximiser l'utilisation d'old Trafford ${ }^{7}$. Les chiffres de dépenses des spectateurs les jours de match feront, à ne pas douter, rêver les dirigeants 
de clubs français. Lors de la saison 2003-2004, les revenus de jour de match atteignent la somme de 88,3 millions d'euros, soit $36 \%$ du chiffre d'affaires. Une année auparavant, le chiffre est encore plus impressionnant. Les spectateurs ont rapporté la somme de 101,9 millions euros, soit $41 \%$ du chiffre d'affaires. Comment peut-on expliquer cette formidable réussite? Certainement pas avec l'unique regard d'un économiste. Suivons de près les charges émotionnelles que recouvre la marque Manchester United.

\section{Le produit Manchester United : objet de consommation et de reconnaissance}

6 L'une des raisons de l'impressionnante prospérité des secteurs commerciaux se trouve dans les représentations qui entourent le produit Manchester United. «Il ne suffit pas de vendre un objet, il faut lui donner du sens, surtout quelque chose qui permette aux fans de se reconnaître, de s'identifier à Manchester United» nous confie Steve Richards, directeur du secteur merchandising ${ }^{8}$. Le développement des activités économiques, dans lesquelles les supporters se retrouvent et se reconnaissent, s'accélère depuis ces dix dernières années. objectivement parlant, Manchester United est véritablement un modèle de réussite à la fois local et global. L'objectif des dirigeants s'inscrit dans la logique d'agrandissement de la communauté Manchester United, notamment à l'échelle mondiale. Plusieurs procédés sont utilisés pour animer des voies d'affiliation au club, quel que soit l'endroit où se trouve le supporter. La diffusion d'un magazine, la nomination du responsable du département international, une chaîne de télévision et un musée apparaissent comme des productions très parlantes.

7 L'envergure économique internationale commence dans la création d'un magazine. C'est en 1992 que sort le premier numéro de Manchester United Magazine (rebaptisé United en 2001). Sa particularité est le fait qu'il soit vendu dans plus de vingt cinq pays, et surtout qu'il soit traduit en norvégien, thaïlandais ou en cantonnais pour les fidèles supporters installés loin de Manchester. D'un point de vue économique, le mensuel est le magazine sportif au plus gros tirage (plus de 200000 exemplaires), et aussi le mieux vendu dans le Royaume Uni. A l'intérieur, on y découvre toute l'actualité interne du club. Le supporter australien lira par exemple l'interview d'un nouveau joueur, le récit d'une rencontre mémorable racontée par un ancien joueur, le compte rendu de la tournée estivale qui a eu lieu dans son pays ou encore les impressions de fidèles supporters à Hong Kong réunis dans une des associations officielles de supporters un jour de rencontre. Pour les dirigeants, le magazine constitue une vitrine officielle du club. Pour certains supporters, le mensuel est un moyen privilégié de se rapprocher de Manchester United, en découvrant par exemple les facettes de la vie d'un joueur et, ce qui revient très souvent, l'histoire de son attachement à Manchester United. L'année 1998 connaît une accélération des produits d'affiliation.

8 Manchester United International Ltd est fondé. Le département à la charge des relations extérieures, notamment du rayonnement international. En retour du fort soutien des supporters scandinaves, les dirigeants nomment à la tête de l'organisation, Vange Kourentis, originaire de Norvège. Depuis 1981, c'est le lieu où sont centralisées les branches de supporters des pays scandinaves. C'est un pays qui suit de près l'actualité footballistique anglaise, puisque les rencontres de la Premier League sont retransmises par la chaîne BSkyB. Les réussites des internationaux Ronny Johnsen, Henning Berg, et surtout 
du redoutable avant-centre Ole Gunnar Solskjaer renforcent les liens entre le club et la communauté de supporters de Manchester United de Norvège. En 1999, la branche norvégienne comptait 32000 membres. En août 2002, l'association regroupait 48000 membres, dont 24000 Norvégiens, 18000 Danois et 2000 Suédois. Le document d'information de Manchester United International condense remarquablement les objectifs du département. Parmi les buts soulignés, nous retiendrons ces deux phrases : « Renforcer la fidélité des supporters de longue date; Renforcer l'image de Manchester United comme une marque internationale $»^{9}$. D'une fidélité de supporters locaux, on s'achemine à une fidélité globale.

9 Le lancement de MUTV (Manchester United Television), en collaboration avec la chaîne câblée BSkyB du magnat international de la presse Rupert Murdoch, accentue la visibilité mondiale. Le club dispose d'un media unique et exclusif pour renforcer les croyances mythologiques autour de son histoire ou de ses joueurs de légendes. Du mardi 17 heures au dimanche minuit, quinze émissions sont présentées. Le passionné de Manchester United trouvera dans les différents programmes ce qui le lie avec le club. Tout un ensemble d'émissions permet de nourrir l'appétit de chaque fan. Les rencontres mémorables d'anciennes légendes telles que George Best ou Eric Cantona, les matchs de la saison courante, l'entretien exclusif de stars actuelles, Ryan Giggs ou Wayne Rooney, les confidences d'avant match de l'entraîneur Sir Alex Ferguson, les avis des supporters sur les rencontres ou sur les questions brûlantes (hausse des prix de carte d'abonnement, politique sécuritaire, politique de répartition des places), les opinions d'anciennes gloires, la rencontre de futurs talents des équipes de jeunes garantissent à MUTV une relation singulière avec les supporters-spectateurs de Manchester United. Comme le souligne le slogan du prospectus (Bring United home!) ${ }^{10}$, l'actualité de Manchester United arrive directement dans chaque foyer de supporter. Par ailleurs, le site Web, créé en 1998, connaît un succès fulgurant. En 2004, il était visité chaque mois par 13 millions d'internautes. La fidélité de l'assistance aux rencontres à Old Trafford se complète par l'assistance de rencontres à domicile.

Enfin, le musée constitue symboliquement le lieu le plus significatif du poids émotionnel qui entoure les édifices de grande rentabilité économique. Construit en 1986, le musée est ensuite déplacé sous l'immense tribune Nord. Il apparaît complètement remodelé et agrandi lors de son inauguration en 1998, par le célèbre footballeur brésilien, Pelé. La visite du musée à trois niveaux offre aux supporters ou aux touristes l'occasion de suivre plus d'un siècle d'histoire de Manchester United. Bien évidemment, la réussite sportive est soigneusement présentée à travers les nombreux trophées nationaux (FA. Cup, Premier League, Charity Cup) et internationaux (Coupe d'Europe des Clubs Champions, Coupe d'Europe des Vainqueurs de Coupe, Coupe Intercontinentale) qui luisent derrière les vitrines (Trophy Room). Un tour est organisé à l'intérieur des vestiaires où le guide pointe les places respectives des joueurs et livre quelques confidences des rituels de certains. On apprend que tel joueur préfère s'asseoir avec untel par superstition, que les joueurs mangent, à la mi-temps, des biscuits Jaffa fourrés à l'orange (un des sponsors du club). On découvre la salle et les sièges où Sir Alex Ferguson reçoit l'entraîneur adverse avant et après chaque rencontre. Une salle est entièrement dédiée aux supporters. Plusieurs objets de représentations (blousons, écharpes, disques, photos), des portraits pris le jour d'un match montrant des visages passionnés, et des récits de trajet de supporters d'origines géographiques et ethniques variées permettent de reconnaître et de valoriser la communauté de fans de Manchester United. Les entretiens que nous avons effectués avec 
les supporters mettent en avant la section dédiée à la tragédie de Munich, du 6 février 1958, où vingt trois personnes, dont huit joueurs, ont trouvé la mort. On y découvre le télégramme d'un joueur à sa logeuse quelques heures avant l'accident. Les extraits de journaux et des documents sonores de l'époque font revivre le drame. Les messages de condoléances reçus de divers pays accentuent le caractère international du drame. Pour de nombreux fans, c'est la partie la plus touchante de la visite. Le musée va constituer un lieu de mémoire collective pour de nombreux supporters. Depuis 1987, l'endroit est l'un des lieux les plus fréquentés de Manchester, quelquefois loin devant de prestigieux musées. Le chiffre des entrées est fulgurant. En 1999, on dénombre 273889 entrées. En 2004, il y eut 232831 visiteurs $^{11}$. Pour donner au musée un caractère social et une dimension locale, un département éducatif anime plusieurs ateliers de citoyenneté et de savoir-vivre. Les jeunes adolescents des collèges de Manchester et ses alentours, apprennent par exemple, l'histoire sociale de Manchester, intègrent les principes du fair play, contribuent aux campagnes contre le racisme, et pour l'égalité des sexes, sur le respect de l'environnement et la nécessité d'une alimentation équilibrée pour lutter contre l'obésité.

\section{Les supporters : un soutien sans limite?}

11 Quand on observe l'histoire des partisans de Manchester United depuis la fin de la seconde Guerre Mondiale, on est tout de suite marqué par l'impressionnante fidélité des fans à Old Trafford, et la force de son expansion internationale. Depuis 1945, Manchester United est le club le plus soutenu d'Angleterre. Les crises d'assistance de la période d'austérité économique (1951-1966), et de la poussée de la violence dans les stades (1974-1984) n'ont guère atténué la moyenne relativement élevée des spectateurs. Si l'on regarde de près les saisons 1956-1957 et 1961-1962, dans lesquelles les moyennes nationales sont les plus basses avec respectivement 29877 et 26106 spectateurs, les Reds Devils conservent un nombre de supporters relativement important. Ce sont en moyenne 45192 et 33490 personnes qui ont assisté aux matchs à Old Trafford. Les raisons de cette résistance des supporters proviennent certainement, d'une adhésion de la philosophie du jeu offensif prônée par l'entraîneur écossais, Matt Busby, alors que de nombreuses équipes appliquent des schémas ultra défensifs importés du continent, en particulier d'Italie. Quelques années plus tard, la relégation en seconde division en 1974, à la suite du départ de joueurs exceptionnels, a peu affecté l'engouement des spectateurs.

De nos jours, l'évolution des recettes de matchs, des cartes d'abonnement annuel, du nombre de membres officiels et des associations de supporters dans le monde permet de parler d'un véritable empire de fidèles supporters. Choisissons les chiffres des membres et des associations officielles. De la saison de son installation en 1987-1988 jusqu'à la fin du championnat 2003-2004, le nombre de membres passe de 40000 à 193000. Le chiffre aurait pu être plus élevé, nous confiait Barry Moorhouse (responsable du département des supporters) ${ }^{12}$, mais les administrateurs ont instauré un numerus clausus de garantie en 1996, afin de ne pas décevoir les fans de plus en plus nombreux, désireux d'être membre pour postuler à une carte d'abonnement annuel. En effet, le nombre de places au stade étant limité à 67800 , il serait déraisonnable d'accueillir un nombre illimité de membres.

L'autre singularité de la puissance du soutien porte sur les associations. En 1989, on dénombre 63 branches. En 2004, le chiffre a plus que triplé, puisqu'on enregistre 231 branches. Les deux Irlande concentrent la majorité des associations ( 86 branches). Là-bas, 
les sections de supporters sont plus importantes que celles localisées à Manchester, et sa banlieue (9 branches). Les liens historiques entre Manchester et Belfast, et surtout les carrières exceptionnelles de Georges Best, Norman Whiteside, Roy Keane expliquent la popularité de Manchester United. L'étranger contribue à étendre la popularité du club puisque l'on dénombre 25 branches, dont dix en Europe (Allemagne, Belgique, Suisse, Norvège) ${ }^{13}$ et six dans l'hémisphère Sud (Australie, Nouvelle Zélande, Singapour). L'explosion des branches de supporters et les politiques de fidélisation orchestrées par les administrateurs ont bouleversé la position et le profil sociologique des supporters. Deux nouvelles générations de supporters se révèlent intéressantes à suivre. Il s'agit de ceux que nous pouvons identifier comme les principaux consommateurs, les plus fidèles clients du produit Manchester United. D'un autre côté, d'autres se singularisent par leur investissement en tant que groupe de pression, et comme des acteurs incontournables. Ils sont décidés à jouer un rôle prépondérant dans l'organisation, notamment par le refus sans condition à l'arrivée de puissants hommes d'affaires dans le destin du club, au nom d'une certaine tradition à préserver.

Les supporters-clients sont dotés d'un pouvoir d'achat élevé. Ils participent activement à la puissance économique. Leur investissement à Manchester United est pour certains relativement récent. Un businessman gallois nous livre qu'il supporte Manchester United depuis une dizaine d'années. Un médecin de Birmingham suit le club depuis peu. C'est le charisme d'Eric Cantona qui l'a séduit. Un autre homme d'affaires irlandais venu de Belfast avoue qu'il supporte les Reds depuis le début des années 1990, période où l'équipe commence à faire de l'Irlande du Nord un lieu de matches de pré-saison. La carte d'abonnement annuel, les dépenses de match (pub, frais d'hôtel, taxi), l'achat du programme de match pour des amis ou pour soi, afin de constituer une bibliothèque d'objets de mémoire constituent quelques signes de leur attachement à Manchester United. Certains n'hésitent pas à suivre le club lors de rencontres importantes contre Liverpool ou Arsenal. D'autres effectuent les déplacements à l'étranger, notamment lors de confrontations contre de prestigieuses équipes européennes (Real de Madrid, Juventus de Turin, Bayern de Munich). Ils effectuent les voyages par le biais du département commercial qui organise les Executive trips (séjours de luxe). On peut les apercevoir dans les nombreuses loges vitrées qui entourent le stade. La catégorie des classes moyennes (avocat, architecte, patrons de commerce) représente une part importante. Cependant, on trouve aussi une frange de la petite bourgeoisie (enseignant, informaticien, employé de banque) qui déclare faire d'énormes sacrifices pour satisfaire leur principal loisir. Celle-ci habite majoritairement dans l'agglomération de Manchester. Ces personnes se distinguent également par une considération des activités sociales du club. Certaines ont vu d'un bon œil le partenariat entre Manchester United et l'organisation caritative mondialement réputée, Unicef, en 1999. La charte du club lancée en juillet 2002, où les devoirs et les obligations des administrateurs vis-à-vis des supporters sont stipulés, marque un moment décisif. Les dirigeants ne peuvent ignorer les attentes de groupes de supporters concernés par les domaines qui dépassent le cadre footballistique, et également préoccupés par ce que peut exiger un client fidèle à une entreprise lucrative. Une partie des supporters va pointer la relative sous-représentation des fans au sein de l'organisation et se constituer comme un groupe de pression.

Les supporters-acteurs exigent une reconnaissance de la part des administrateurs. La fondation d'IMUSA (Independent Manchester United Supporters Association) en 1995 traduit une nouvelle mouvance dans le sentiment de supportérisme. La campagne contre la 
décision des directeurs d'accepter l'offre de rachat du club par Rupert Murdoch a été significative ${ }^{14}$. Elle a véritablement permis de percevoir les supporters autrement, notamment comme des acteurs engagés et non plus comme de simples consommateurs. Cette campagne a aussi permis à une partie des supporters d'obtenir une reconnaissance médiatique à partir d'un engagement de passionnés de Manchester United et d'individus concernés par l'avenir du football en général. Du 6 septembre 1998 au 9 avril 1999, les membres d'IMUSA s'opposent fermement à l'arrivée du puissant magnat de la presse. Pendant plusieurs mois, ces supporters se battent pour des idéaux précis : l'identité de Manchester United et la reconnaissance d'une position valorisée dans le développement du club. Le 15 septembre 1998, l'immense et luxueuse salle de concert classique, Bridgewater Hall, sert de lieu de ralliement pour débattre de l'avenir de Manchester United. Pendant quelques heures, ils saisissent l'opportunité de signifier le poids des supporters dans l'organisation du club. Certains rappellent que, sans les supporters, le club n'a pas lieu d'être. D'autres s'enorgueillissent de la puissance économique de leur fidélité et de leur amour pour Manchester United. Un voisin déclare « Manchester United, c'est nous. Demander à Murdoch où il était quand nous étions en seconde division, il y a quelques années. Le moteur de Manchester United, c'est les supporters $»^{15}$. Ce sont des universitaires, des journalistes réputés, l'ancien batteur du groupe Queen, des étudiants, des avocats, des hommes d'affaires locaux, des employés de bureau qui réussirent, sous l'aval du gouvernement, en particulier de la commission du Monopole et de la Concurrence, à faire échouer les appétits économiques de Rupert Murdoch, et à calmer les ardeurs de grandeur des administrateurs. La retenue des dirigeants face aux incessantes offres de rachat du club par le milliardaire américain Malcom Glazer ${ }^{16}$ ces derniers mois est en quelque sorte une preuve du poids des supporters acteurs. Cependant, il faut se garder d'une catégorisation dualiste, et dès lors trop réductrice. Un point unit l'ensemble des supporters, il s'agit des trajets d'appartenance à une véritable communauté Red Devils. L'ambition des administrateurs est de faire de Manchester United, une institution renommée pour ses exploits sportifs, mais également pour ses exceptionnelles réussites commerciales. La conversion du supporter en véritable consommateur fidèle s'insère dans cette logique sportive et économique. Du maintien des succès sportifs et de la valorisation des supporters dépend l'avenir des politiques de fidélisation.

\section{BIBLIOGRAPHIE}

Boli C : Manchester United, l'invention d'un club. Deux siècles de métamorphoses, Paris, La Martinière, 2004.

Hamil S, Michie J, et Oughton C: The business of football. A game of two halves, Londres, Mainstream, 1999.

Russell D: Football and the English, Preston, Carnegie, 1997.

Williams J: Is it all over? Can football survive the Premier League?, Reading, South Street Press, 1999. 


\section{NOTES}

1. Thiriez $\mathrm{F}$, «Les clubs français à l'épreuve du «foot-business» ", in Pouvoirs, Revue Française d'Etudes Constitutionnelles et Politiques, Le Football, n 101, avril. 2002, pp. 65-74.

2. Cf. Boli C: Manchester United, l'invention d'un club. Deux siècles de métamorphoses, Paris, La Martinière, 2004.

3. Manchester United Development Association, Football Pool Rules, $n^{\circ} 1,1961$.

4. Entretien avec Edward Freedman, 20 novembre 1996.

5. Le stade est la propriété de Manchester United depuis 1927.

6. Deloitte \& Touche, England's Premier Clubs, avril 2001, p. 31.

7. Manchester United Annual Report 2004, p. 4.

8. Entretien avec Steve Richards, 2 septembre 1998.

9. Prospectus de Manchester United International, octobre 1999, p. 3.

10. Prospectus de MUTV, juillet 2004, office du tourisme de Manchester.

11. Manchester United Archives Department.

12. Entretien réalisé le 4 novembre 1996.

13. En mars 2003, une branche française se forme. Mais, celle-ci n'apparaît pas dans la liste des branches officielles du livret des supporters. Manchester United Official Yearbook, 2003-2004, pp. 216-224.

14. Brown A-Walsh A, Not for sale. Manchester United, Murdoch and the defeat of BskyB, Londres, Mainstream, 1999.

15. :Boli C, Manchester United, L'invention d'un club. Deux siècles de métamorphoses, Paris, La Martinière, 2004, p. 348.

16. La Tribune, lundi 7 février 2005, p. 31. L'absence de titre prestigieux (Coupe d'Europe des Clubs Champions), la baisse du chiffre d'affaires pour la seconde année consécutive, et l'attrait de Chelsea depuis la reprise du milliardaire russe Roman Abramovich ont certainement motivé les membres du conseil administratif d'accepter l'offre de Malcom Glazer. Ce dernier est devenu l'actionnaire majoritaire avec $78,1 \%$ des parts, à contre cœur de certains supporters. The Guardian, vendredi 13 mai 2005, pp. 22-24.

\section{RÉSUMÉS}

Depuis 1961, l'exceptionnelle réussite économique de Manchester a est largement liée à une politique de fidélisation des supporters. Les mutations organisationnelles entamées par le club à partir de 1991 ont pour effet de transformer les supporters en véritables consommateurs. Certains vont s'affirmer comme des acteurs décisifs par un engagement très actif dans plusieurs causes, dont la campagne anti-Murdoch.

Since 1961, the unique financial success of Manchester United is largely based on supporters loyalty policy. By 1991, the changing structure has transformed the supporters into customers. Nevertheless, some of them will express themselves as prominent leaders, engaging themselves in various issues, such as the campaign against Murdorch. 


\section{AUTEUR}

\section{CLAUDE BOLI}

Claude Boli est docteur en histoire (De Montfort University, Leicester, Angleterre), et en sociologie (Université de Nantes). Ses travaux portent sur l'impact social du football professionnel en Angleterre depuis la fin du XIX siècle. Il enseigne l'histoire et la sociologie à l'Université d'Evry (Dept. STAPS), et est chercheur associé à l'International Centre for Sport History and Culture, Leicester. Auteur de : Manchester United, l'invention d'un club. Deux siècles de métamorphoses, Paris, La Martinière, 2004. 